\title{
Neurophotonics
}

\section{Single-cell imaging tools for brain energy metabolism: a review}

Alejandro San Martín

Tamara Sotelo-Hitschfeld

Rodrigo Lerchundi

Ignacio Fernández-Moncada

Sebastian Ceballo

Rocío Valdebenito

Felipe Baeza-Lehnert

Karin Alegría

Yasna Contreras-Baeza

Pamela Garrido-Gerter

Ignacio Romero-Gómez

L. Felipe Barros 


\title{
Single-cell imaging tools for brain energy metabolism: a review
}

\author{
Alejandro San Martín, \\ Sebastian Ceballo, ${ }^{a}$ Rocío Valdebenito, ${ }^{a}$ Felipe Baeza-Lehnert, ${ }^{a}$ Karin Alegría, ${ }^{a}$ Yasna Contreras-Baeza, ${ }^{\text {a,b }}$ \\ Pamela Garrido-Gerter, ${ }^{\mathrm{a}, \mathrm{b}}$ Ignacio Romero-Gómez, ${ }^{\mathrm{a}, \mathrm{b}}$ and L. Felipe Barros ${ }^{\mathrm{a}, \star}$ \\ ${ }^{a}$ Centro de Estudios Científicos, Arturo Prat 514, Valdivia, 5110466, Chile \\ buniversidad Austral de Chile, Valdivia, Chile
}

\begin{abstract}
Neurophotonics comes to light at a time in which advances in microscopy and improved calcium reporters are paving the way toward high-resolution functional mapping of the brain. This review relates to a parallel revolution in metabolism. We argue that metabolism needs to be approached both in vitro and in vivo, and that it does not just exist as a low-level platform but is also a relevant player in information processing. In recent years, genetically encoded fluorescent nanosensors have been introduced to measure glucose, glutamate, ATP, NADH, lactate, and pyruvate in mammalian cells. Reporting relative metabolite levels, absolute concentrations, and metabolic fluxes, these sensors are instrumental for the discovery of new molecular mechanisms. Sensors continue to be developed, which together with a continued improvement in protein expression strategies and new imaging technologies, herald an exciting era of high-resolution characterization of metabolism in the brain and other organs. ( The Authors. Published by SPIE under a Creative Commons Attribution 3.0 Unported License. Distribution or reproduction of this work in whole or in part requires full attribution of the original publication, including its DOI. [DOI: 10.1117/1 .NPh.1.1.011004]
\end{abstract}

Keywords: membrane transport; glycolysis; mitochondria; metabolic flux; optogenetics; Förster resonance energy transfer. Paper 14008VSSR received Feb. 15, 2014; revised manuscript received Apr. 9, 2014; accepted for publication Apr. 10, 2014; published online May 29, 2014.

\section{Metabolism and Neuroscience}

The metabolic core of a neuron is similar to that of any other cell, so metabolism is seen as a platform for the unique functions of the brain, with emergent processes such as synaptic transmission, memory, and cognition. According to this view, the brain needs metabolism in the same manner a concerto requires instruments. Even so, it is not the quality of the instruments that makes a concerto unique, but the score or the high-level design, metabolism is not perceived as a major part of what makes the brain unique.

However, the musical analogy does not consider that nervous cells have continued to evolve throughout the process of encephalization, fostered by the allopatric influence of the blood-brain barrier. While the same bits and pieces are being used, the brain has developed its own set of metabolic rules. For example, glutamate, a humble metabolite elsewhere, doubles in the brain as an excitatory neurotransmitter. How are these roles reconciled? Another novelty was the advent of glia, bringing division of labor and a new modulatory level for information processing. Astrocytic glycogen, through lactate production, is involved in memory processing. ${ }^{1-3}$ Astrocytes regulate the extracellular concentration of virtually every molecule in the brain interstitium, including glutamate and $\mathrm{K}^{+}$, integrating information from thousands of individual synapses, and they release metabolites such as purines and lactate, which affect the excitability of adjacent neurons. ${ }^{4-7}$ Thus, lactate is now regarded as both a fuel and a bona fide signaling molecule. The mismatch between glycolysis and mitochondrial oxidation that determines net lactate production is observed throughout the brain during childhood

*Address all correspondence to: L. Felipe Barros, E-mail: fbarros@cecs.cl but becomes restricted in the adult brain to areas expressing neotenous genes associated with synaptic remodeling. ${ }^{8}$ These tonically active, lactate-rich areas, collectively known as the default mode network, ${ }^{9}$ are most vulnerable to amyloid deposition. ${ }^{10,11}$ Other evidence for a higher role for metabolism relates to energy. Whereas brain tissue is expensive, consuming fuel 10 times faster than the rest of the body, the extraordinary growth of the brain during mammalian evolution took place in a context of chronic food shortage. ${ }^{12}$ Therefore, evolutionary strategies built around energy constraints are expected to be part of the brain's blueprint. The above examples suggest that metabolism exists not just as a low-level platform, but as a relevant player in information processing. Metabolism is also interesting for practical reasons because the study of cognition in health and disease relies heavily on fluorodeoxyglucose-positron emission tomography (FDG-PET) scanning and functional magnetic resonance imaging (fMRI), techniques that monitor local energy metabolism. Moreover, defective metabolism can be troublesome, and a promising line of research on neurodegeneration has been inspired by the finding of metabolic alterations decades before the onset of clinical symptoms. ${ }^{10,11}$

\section{Metabolism Is Modular and Hierarchical}

Metabolism is the sum of the chemical processes that occur in living organisms. Defined in a broader way, it also includes the transport of the chemicals between membrane compartments. Metabolism is hierarchically ordered, spanning multiple spatial and temporal scales, and is modular, hence accessible to reductionist investigation ${ }^{13}$ (Fig. 1). The modular nature of metabolism is explained by a progressive weakening of interactions as distances become larger. Molecular processes are dominated by strong, short-range forces, and are relatively insensitive to the 


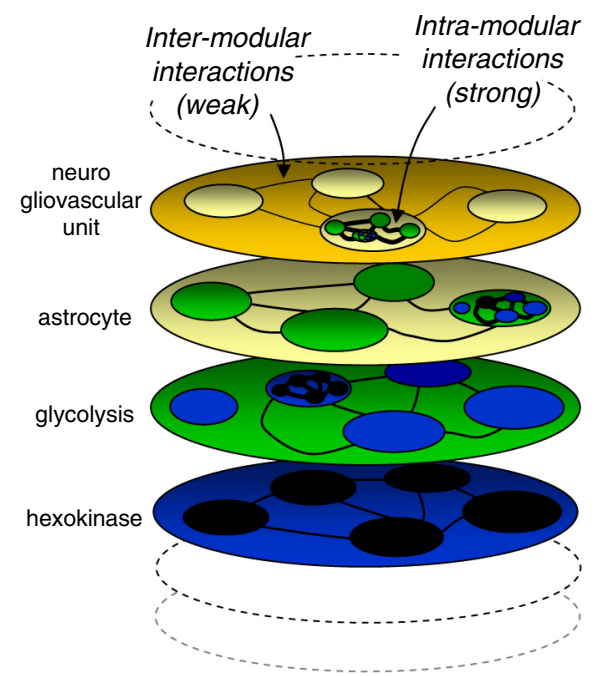

Fig. 1 Organization of metabolism. The living organism is represented as a hierarchical stack of modular systems. Interactions at any level are stronger than at higher levels, which is the basis for dissectability. In this example regarding metabolism, the enzyme hexokinase (represented by a blue oval) is shown to be constituted by amino acids (black ovals). In the next level up, hexokinase associates with other enzymes and cofactors to constitute the glycolytic machinery (green oval). Further up the organizational ladder, the glycolytic machinery associates with organelles and other structures to form an astrocyte (yellow oval), which in turn interacts with neurons and vessels to shape the neurogliovascular unit (orange oval) [after J. G. Miller's generalized living system $\left.{ }^{13}\right]$.

weaker, long-range forces that determine the structure of cells and tissues. Thus, microscopic properties of enzymes, such as substrate specificity, withstanding harsh extraction and purification procedures, are what proved fundamental for the mapping of the metabolic network during the first half of the 20th century. At the next level of organization, enzymes associate with other proteins and lipids, interactions that are important for regulation. Except for the inner workings of mitochondria, which are readily purified and studied by respirometry, the subcellular level of metabolic organization is virtually uncharted territory. For instance, there is evidence of physical interaction and functional cross-talk between the glycolytic machinery and the $\mathrm{Na}^{+} / \mathrm{K}^{+}$ATPase, the great energy spender, but the nature of the interaction does not seem to be energetic. ${ }^{14}$ What are the mechanisms that match energy production to energy consumption? The enzymes of glycolysis are expressed in stoichiometric proportions. ${ }^{15}$ Is this finding related to their possible association in functional modules? Hexokinase, the first enzyme of glycolysis, is bound to mitochondria and also to the $\mathrm{Na}^{+}$/glutamate co-transporter. ${ }^{16,17}$ Are these interactions part of the machinery matching glycolysis to mitochondrial respiration? Mitochondrial metabolism is sensitive to calcium signals. ${ }^{18-20}$ What are the relative roles of energy status, redox status, substrate supply, and calcium-signaling in the control of mitochondrial flux? Are mitochondria fueled by pyruvate or by lactate? ${ }^{21}$ How can glycogen be mobilized without generating a traffic jam at phosphofructokinase? These are the kinds of questions that need to be tackled at the subcellular level.

Metabolic organization at the cellular level presents further unresolved issues. In culture and in acute brain slices, astrocytes are more glycolytic than neurons. ${ }^{22,23}$ Consistently, immunohistochemistry and mRNA measurement of fluorescence-activated cell sorting-isolated brain cells show higher expression of glycolytic and biosynthetic enzymes in astrocytes than in neurons, ${ }^{24,25}$ a divergence further enhanced by posttranslational regulation of flux-controlling enzymes. ${ }^{26-28}$ The study of brain cells in culture has revealed specific regulatory modules that operate on membrane transport, glycolysis, and mitochondria, some of them in response to signals of neuronal activity like glutamate and $\mathrm{K}^{+} .{ }^{7,29-33}$ Cultured cells, which provide optimum control of experimental variables, are still an indispensable tool for the discovery of subcellular and cellular mechanisms. ${ }^{34}$ But the parameter space is more restricted in situ than in culture, and new approaches that are able to probe metabolism at cellular resolution, in tissue slices and in living animals, are required to gauge the pertinence of the molecular mechanisms that are being discovered. There are important questions that need to be addressed through in vivo experiments, such as the concentrations of glucose and lactate in astrocytes, neurons, and oligodendrocytes, the extent and direction of glucose, lactate and pyruvate fluxes within and between cells, how the brain tissue copes with the large changes in local energy demand that accompany neural activity, the metabolism of subtypes of cells, and the metabolism of the diseased brain.

\section{High-Resolution Imaging of Metabolism}

Metabolic processes span many orders of spatiotemporal magnitude, from submillisecond nanometer enzyme catalysis to the years that it takes to deposit an amyloid plaque. One technique that may cover the full range of metabolism is fluorescence microscopy. Wide-field, confocal, multiphoton, light-sheet, super-resolution and other microscope systems need to be combined with a toolkit of fluorescent probes to make metabolism visible in the way calcium dyes do for neuronal activity. So far metabolism has been imaged using $\mathrm{NAD}(\mathrm{P}) \mathrm{H}$ and flavoprotein autofluorescence, fluorescently labeled metabolites, and, more recently, with genetically encoded nanosensors. Fast fluctuations in the autofluorescence of brain tissue in acute slices and in vivo have revealed that metabolism can be as dynamic as intracellular calcium. ${ }^{35-39}$ The extremely rapid response of NADH is consistent with its turnover time, which in brain cells is in the order of a few milliseconds. ${ }^{40}$ However, the interpretation of autofluorescence data is limited by several factors, including uncertainty about the relative weight of free and protein-bound $\mathrm{NADH}$, interference from NADPH, which in the cytosol is much more abundant, difficulties in assigning the signals to specific subcellular compartments, and the inherent ambiguity of concentration with respect to flux, compounded by the requirement for UV light, which is toxic and unavailable in standard confocal microscopes.

An alternative approach is the use of fluorescently tagged metabolites, ${ }^{41}$ as was done with glucose and its fluorescent analogues. They provide good signal-to-noise ratio, but because the tag interferes with the biochemical function of the metabolite, these compounds may only be used as tracers. For example, in the fluorescent glucose analogs 2-(N-(7-nitrobenz-2-oxa1,3-diazol-4-yl)amino)-2-deoxy-glucose (2-NBD-glucose) and 6-NBD-glucose, the fluorescent group is hydrophobic, which enhances binding to the glucose transporter, severely slowing down membrane translocation. ${ }^{42}$ In this particular case, the slowness of uptake is an advantage, as it provides an extended time window for experimental intervention. With these tools, it has been shown that astrocytic glucose transport is rapidly 
stimulated in an activity-dependent fashion in vitro and in vivo ${ }^{43-46}$ and that metabolite flux through gap junctions may sustain synaptic activity. ${ }^{47}$ Once inside cells, 2-NBDG is slowly phosphorylated by hexokinase and trapped as 2-NBDG-P, a readout of the glycolytic rate that becomes accessible after free tracer wash-out. Application of 2- and 6-NBDG protocols to acute slices prepared from cerebellum and hippocampus showed that astrocytes and Bergmann glia are several-fold faster at transporting and metabolizing glucose than neurons, ${ }^{23,48}$ consistent with constitutive lactate shuttling from glia to neurons. ${ }^{49,50}$ As its accumulation is limited by transport and not by metabolism, 2-NBDG uptake does not provide sufficient temporal resolution for investigation of the rapid events underlying fMRI or the rapid fluctuations detected by real-time measurement of tissue autofluorescence, oxygen, and lactate.

The most recent addition to the metabolic imaging toolkit is the genetically encoded nanosensor. Typically, a nanosensor is a fusion protein comprising a bacterial protein that binds a specific metabolite, and one or two fluorescent proteins. Binding of the metabolite to the bacterial moiety leads to a conformational change that modifies the physical properties of the fluorescent partner(s). Some sensors have two fluorescent proteins with overlapping emission and excitation spectra, capable of

Table 1 Genetically encoded sensors for molecules involved on brain energy metabolism.

\begin{tabular}{|c|c|c|c|c|c|c|c|c|}
\hline Sensor & $\begin{array}{c}\text { Original } \\
\text { description }\end{array}$ & $\begin{array}{l}\text { Addgene } \\
\text { number }\end{array}$ & $\begin{array}{c}\text { Ligand } \\
\text { binding moiety }\end{array}$ & Readout & $\begin{array}{l}\text { Maximum } \\
\text { change (\%) }\end{array}$ & $\begin{array}{c}\text { Type of } \\
\text { measurement }\end{array}$ & Application & Users \\
\hline \multicolumn{9}{|l|}{ Glucose } \\
\hline FLIP series & $\begin{array}{c}2003 ; 2008 \\
(52,53)\end{array}$ & 17866 & MglB & $\begin{array}{l}\text { FRET (CFP/ } \\
\text { Citrine) }\end{array}$ & 60 & Quantitative & $\begin{array}{l}\text { Relative level } \\
\text { Concentration } \\
\text { Glut activity } \\
\text { Glycolytic flux }\end{array}$ & $\begin{array}{c}(23,31,40 \\
52-82)\end{array}$ \\
\hline \multicolumn{9}{|l|}{ Glutamate } \\
\hline $\begin{array}{l}\text { FLIPE/ } \\
\text { GluSnFR }\end{array}$ & $\begin{array}{c}2005 ; 2008 \\
(83,84)\end{array}$ & $\begin{array}{l}13541 \\
13545\end{array}$ & YbeJ & $\begin{array}{l}\text { FRET (CFP/ } \\
\text { Citrine) }\end{array}$ & 44 & Quantitative & $\begin{array}{l}\text { Relative level } \\
\text { Concentration }\end{array}$ & (83-88) \\
\hline iGluSnFR & $\begin{array}{c}2013 \\
(89)\end{array}$ & $\begin{array}{l}41732 \\
41733\end{array}$ & YbeJ & $\begin{array}{l}\text { Intensity } \\
\text { (cpGFP) }\end{array}$ & 400 & $\begin{array}{c}\text { Semi- } \\
\text { quantitative }\end{array}$ & Relative level & $(89,90)$ \\
\hline \multicolumn{9}{|l|}{ ATP } \\
\hline ATeam & $\begin{array}{c}2009 \\
(91)\end{array}$ & 28003 & $\begin{array}{l}\mathrm{F}_{0} \mathrm{~F}_{1}-\mathrm{ATP} \\
\text { synthase } \\
\varepsilon \text { sub-unit }\end{array}$ & $\begin{array}{l}\text { FRET (mseCFP/ } \\
\text { cpVenus173) }\end{array}$ & $150^{\mathrm{a}}$ & $\begin{array}{c}\text { Semi- } \\
\text { quantitative }\end{array}$ & Relative level & $(58,91-112)$ \\
\hline PercevalHR & $\begin{array}{c}2009 ; 2013 \\
(113,114)\end{array}$ & 49083 & GInK & $\begin{array}{l}\text { Intensity } \\
\text { (cpVenus) }\end{array}$ & $>200^{a}$ & $\begin{array}{c}\text { Semi- } \\
\text { quantitative }\end{array}$ & Relative level & $(113-117)$ \\
\hline \multicolumn{9}{|l|}{ NADH/NAD ${ }^{+}$} \\
\hline Peredox & $\begin{array}{l}2011 \\
(118)\end{array}$ & 32383 & Rex & $\begin{array}{l}\text { Intensity (cpT- } \\
\text { Sapphire) }\end{array}$ & 140 & Quantitative & $\begin{array}{l}\text { Relative ratio } \\
\text { Ratio }\end{array}$ & $(118,119)$ \\
\hline FREX & $\begin{array}{l}2011 \\
(120)\end{array}$ & $\begin{array}{c}\text { Not } \\
\text { available }\end{array}$ & Rex & $\begin{array}{l}\text { Intensity } \\
\text { (cpYFP) }\end{array}$ & 140 & $\begin{array}{c}\text { Semi- } \\
\text { quantitative }\end{array}$ & Relative level & (120) \\
\hline \multicolumn{9}{|l|}{ Lactate } \\
\hline Laconic & $\begin{array}{l}2013 \\
(121)\end{array}$ & $\begin{array}{l}44238 \\
46307\end{array}$ & LIdR & $\begin{array}{l}\text { FRET (mTFP/ } \\
\text { Venus) }\end{array}$ & 38 & Quantitative & $\begin{array}{l}\text { Relative level } \\
\text { Concentration } \\
\text { Production } \\
\text { Consumption } \\
\text { MCT activity }\end{array}$ & $(14,40,121)$ \\
\hline \multicolumn{9}{|l|}{ Pyruvate } \\
\hline Pyronic & $\begin{array}{l}2014 \\
(122)\end{array}$ & 51308 & PdhR & $\begin{array}{l}\text { FRET (mTFP/ } \\
\text { Venus) }\end{array}$ & 40 & Quantitative & $\begin{array}{l}\text { Relative level } \\
\text { Concentration } \\
\text { Mitochondrial } \\
\text { consumption } \\
\text { Glycolytic flux } \\
\text { MCT activity }\end{array}$ & (122) \\
\hline
\end{tabular}

Note: There are six metabolites for which there are optical nanosensors available that have been validated in mammalian cells. The position of these molecules in the metabolic network is indicated in Fig. 3.

a/n vitro measurement of purified protein (data not available for the sensor expressed in cells).

Addgene is a nonprofit plasmid repository (www.addgene.org) 
undergoing Förster resonance energy transfer (FRET), and they respond to the metabolite with a change in FRET efficiency. Being ratiometric, FRET measurements are insensitive to sensor concentration, volume changes, and small focal drifts. Other sensors have only one fluorescent protein, which responds to metabolite binding with a change in emission intensity. Single fluorophore sensors are not intrinsically ratiometric, but a ratio may be obtained by coexpression of a second fluorescent protein. Since the introduction of Cameleon, the first FRET nanosensor in $1997,{ }^{51}$ many probes have been made available to measure ions, enzyme activities, and metabolites in different cell systems from bacteria to whole animals.
Herein we review a restricted set of nanosensors for the six energy metabolites that have so far been validated in mammalian cells: glucose, glutamate, ATP, NADH, lactate, and pyruvate (Table 1). The ligand-binding moiety of these nanosensors is either a periplasmic protein involved in signaling or a transcriptional factor. The first metabolite nanosensors were built with cyan fluorescent protein (CFP) and yellow fluorescent protein (YFP) as an FRET pair, and displayed a small change in fluorescence ratio upon ligand binding $(<10 \%)$. More recent developments include improved variants that are brighter and less $\mathrm{pH}$ sensitive, either from jellyfish or corals, and show a larger dynamic range.
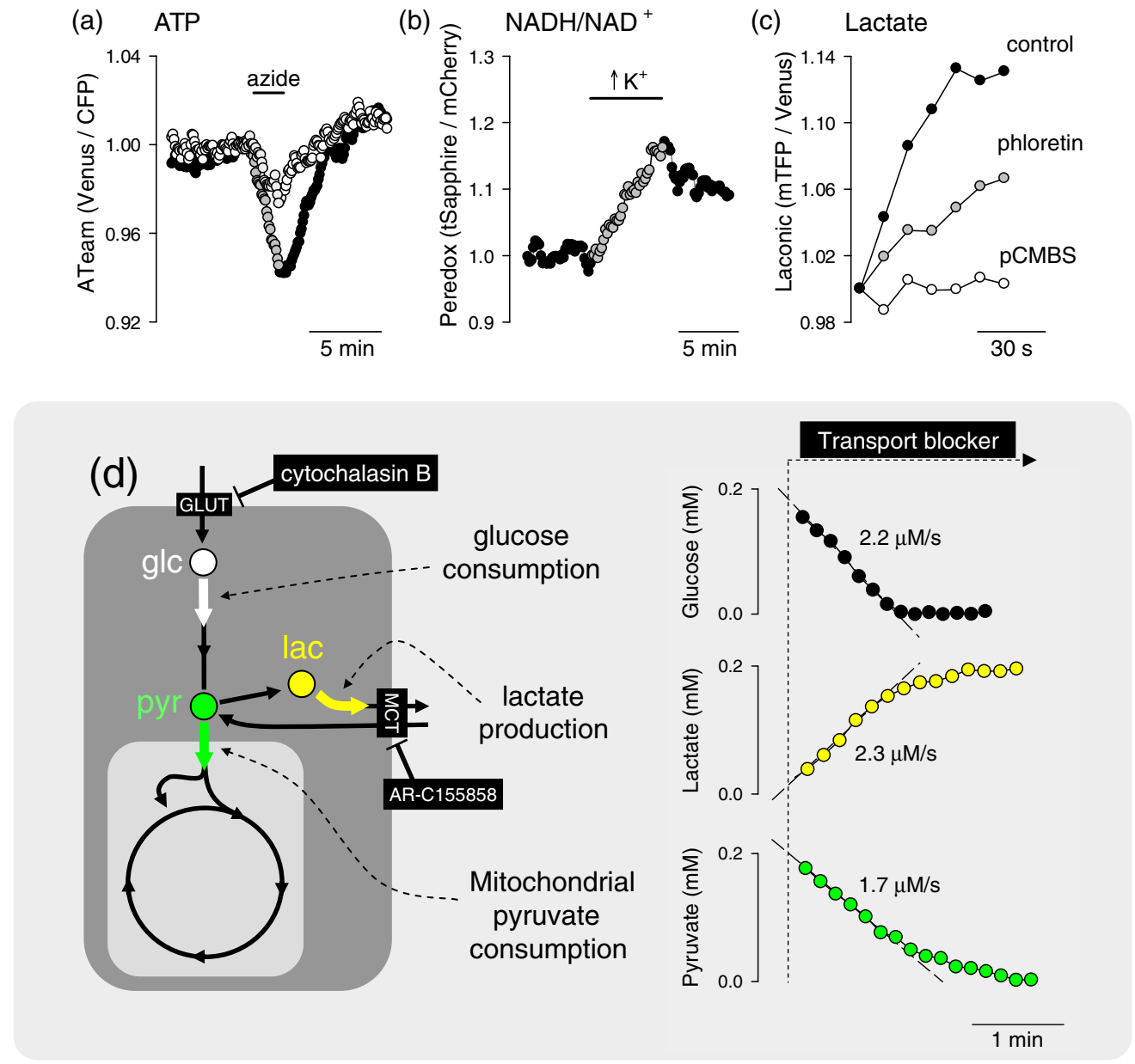

Fig. 2 Examples of the use of genetically encoded nanosensors for the estimation of metabolite levels, concentrations, and fluxes in single cells. (a) Two adjacent astrocytes expressing ATeam $1.03^{91}$ were exposed to the mitochondrial blocker sodium azide $(5 \mathrm{mM})$. Although the metabolic depletion in one of them appears to be stronger, this may not be the case (see text). (b) An astrocyte expressing Peredox ${ }^{118}$ in the cytosol was exposed to a rise in extracellular $\mathrm{K}^{+}$from 3 to $12 \mathrm{mM}$. The observed increase in $\mathrm{NADH} / \mathrm{NAD}^{+}$ratio is consistent with a primary stimulation of glycolytic NADH production, but not with a stimulation of mitochondrial NADH consumption. (c) The uptake of $5 \mathrm{mM}$ lactate was measured in a T98G glioma cell using Laconic ${ }^{121}$ in the absence or presence of the MCT blockers phloretin $(50 \mu \mathrm{M})$ and pCMBS $(500 \mu \mathrm{M})$. The partial inhibitory effect of phloretin becomes evident. (d) Determination of metabolic fluxes with inhibitor-stop protocols. In the steady state, the cytosolic concentrations of glucose, lactate, and pyruvate are constant. Interruption of the steady state with a blocker of the glucose transporter GLUT (cytochalasin B) or of the monocarboxylate transporter MCT (AR-C155858) produces depletion or accumulation at an initial rate equal to the steady-state flux of the pathway. The right panels provide examples of astrocytic glucose consumption estimated with $20 \mu \mathrm{M}$ cytochalasin $\mathrm{B},{ }^{56}$ HEK293 lactate production estimated with $1 \mu \mathrm{M}$ AR-C $155858,{ }^{122}$ and astrocytic pyruvate consumption estimated in the absence of glucose and lactate with $1 \mu \mathrm{M}$ AR-C155858, as detailed in Ref. 122. Rates are indicated. 


\section{Some Applications of the Sensors: Relative Level, Concentration, and Flux}

Much as calcium transients reveal cellular activity, the most frequent use of metabolite nanosensors is for the detection of changes in metabolism. For example, the depletion of glucose observed upon removal of extracellular glucose,${ }^{52}$ the release of glutamate by synaptic activity, ${ }^{83}$ and the depletion of ATP that follows mitochondrial inhibition. ${ }^{91}$ A change in metabolite level during a given experimental challenge shows that metabolism has been altered, but in contrast to calcium transients, the interpretation of metabolite fluctuations may be ambiguous. For instance, Fig. 2(a) illustrates an experiment in astrocytes expressing ATeam, a FRET nanosensor for ATP. Two behaviors were observed in response to oxidative phosphorylation (OXPHOS) inhibition: one cell showed a strong decrease in fluorescence ratio, while another cell in the same field was less affected. This difference could be explained by different changes in ATP concentration, but could also be due to a different resting ATP level. Moreover, even if the resting ATP levels were similar, it is not possible to tell whether the different response is due to (1) mitochondrial function, (2) the capacity of glycolysis to compensate for the loss of ATP production, or (3) perhaps ATP expenditure. Relative levels may be more informative if some complementary information is available. We have reported previously that astrocytes acutely increase their rate of glucose consumption in response to elevated extracellular $\mathrm{K}^{+} .31,59$ In principle, the stimulation may be a "pull" phenomenon, secondary to mitochondrial consumption of cytosolic $\mathrm{NADH}$, as hypothetized for $\mathrm{Ca}^{2+}$-dependent stimulation in neurons, ${ }^{18,20}$ or alternatively, it may be a "push" phenomenon, in which regulation acts on the glycolytic machinery. The $\mathrm{NADH} / \mathrm{NAD}^{+}$nanosensor Peredox provides an answer to this question by showing that $\mathrm{K}^{+}$-stimulated astrocytes acutely increase their cytosolic $\mathrm{NADH} / \mathrm{NAD}^{+}$ratio [Fig. 2(b)], thus evidencing that the primary target of $\mathrm{K}^{+}$is not mitochondrial NADH consumption but the glycolytic machinery. Of note, Peredox provides a better readout than $\mathrm{NAD}(\mathrm{P}) \mathrm{H}$ autofluorescence in terms of specificity and signal-to-noise ratio; it is less phototoxic and offers the possibility of targeted expression, but its temporal resolution is lower (seconds versus milliseconds) and contributes to NADH buffering. It should be borne in mind that the rate at which a given metabolite departs from its steady-state level depends not only on the degree of perturbation but also on the turnover time of the metabolite, i.e., the ratio between concentration and flux through the pathway, which may differ by orders of magnitude along a given pathway. A detailed analysis of this important point and its consequences may be found in Ref. 78. Another use of uncalibrated probes is for the study of membrane transporters. For example, the experiment in Fig. 2(c) shows a glioma cell expressing the lactate sensor Laconic that was sequentially exposed to lactate alone or in the presence of the monocarboxylate transporter (MCT) blockers phloretin or p-chloromercuriphenylsulfonic acid (pCMBS). If rates are compared at similar FRET ratios, it is possible to determine degrees of inhibition, even if the actual concentrations are unknown.

Determination of absolute concentrations can be performed by calibrating the sensor, ideally in the same cell type in which measurements are being made. Full calibration (i.e., determination of a dose-response curve in situ) has been achieved only for the glucose sensor, by blocking glucose consumption while using the glucose transporter or an artificial pore to equilibrate

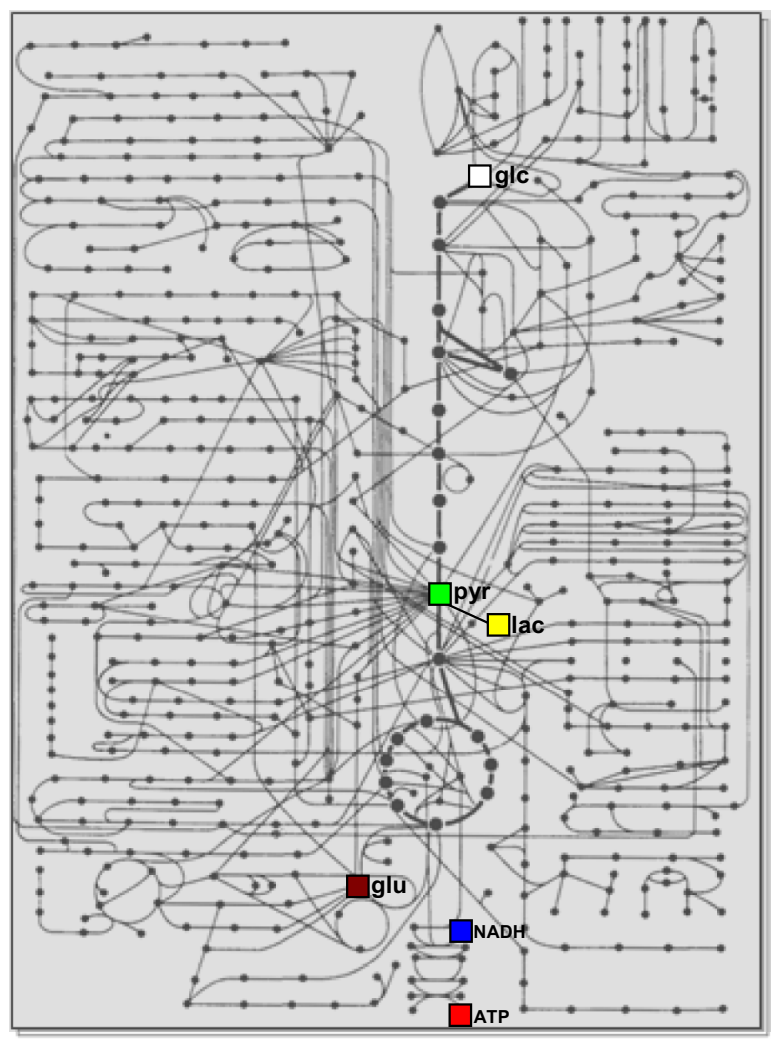

Fig. 3 Mammalian metabolic networks. Schematic representation of mammalian metabolism. Points correspond to metabolites and lines to chemical transformations (from Alberts et al., 1983, cited in Ref. 129). The network location of the six metabolites that have been imaged in mammalian cells is indicated: glucose (glc), pyruvate (pyr), lactate (lac), glutamate (glu), NADH, and ATP.

glucose across the plasma membrane. ${ }^{56,62}$ Detailed protocols have been made available for the use of metabolite sensors in mammalian cells ${ }^{54}$ and more specifically for the use of the glucose sensor. ${ }^{78}$ It is not feasible to halt the metabolism of pyruvate, lactate, or NADH without compromising cell viability, but the corresponding sensors have been two-point calibrated by obtaining readouts at extreme values of the parameters in combination with the affinity constants measured in vitro. ${ }^{118,121,122}$ Thanks to calibration, a glucose gradient was detected across the astrocytic plasma membrane, ${ }^{56,62}$ consistent with a modulatory role for GLUT transporters, and astrocytes were found to have higher $\mathrm{NADH} / \mathrm{NAD}^{+}$ratios than neurons, ${ }^{118}$ supporting the shuttling of redox equivalents between astrocytes and neurons. ${ }^{123}$ Even over a single microscopic field, large differences were detected in glucose concentration and glycolytic flux in adjacent cells, the kind of diversity that may be mined by systems biology approaches, both to extract mechanistic information ${ }^{124}$ or to serve as readouts for single-cell metabolomics. ${ }^{125}$ No calibration is possible for the ATP sensors, so they are considered semiquantitative. A more refined use of the sensors is for the determination of flux through specific pathways. For this purpose, the probe needs to be calibrated, so that the fluorescence ratio is converted into absolute concentration. The strategy for flux determination is to halt flux at a known point in the pathway and then to monitor the rate of accumulation of an upstream metabolite or the rate of depletion of a downstream metabolite. With this 
approach, it has been possible to estimate the rate of glucose consumption ( $\mathrm{FLIP}^{12} \mathrm{glu} 700 \mu \Delta 6$ ), the rates of lactate production or consumption (Laconic), and the rate of mitochondrial pyruvate consumption (Pyronic), as illustrated in Fig. 2(d). Some biological phenomena that have been identified with flux protocols are the regulation of astrocytic glycolysis by high $\mathrm{K}^{+}$and glutamate, ${ }^{31,59}$ feedback control of glycolysis by lactate, ${ }^{65}$ mitochondrial flux in electrically stimulated neurons, ${ }^{122}$ and the Warburg effect in T98G glioma cells. ${ }^{121}$ Using the FRET nanosensors in vivo, Bruno Weber and colleagues have successfully imaged glucose, lactate, and pyruvate in astrocytes and neurons using two-photon microscopy. Practical issues to be addressed for in vivo studies include sensor expression levels, calibration, the metabolic effects of anesthesia, and the adequacy of "inhibitor-stop" protocols for flux determination.

On a cautionary tone, it is important to keep in mind that each nanosensor has its own limitations. A common one affecting selectivity is sensitivity to $\mathrm{pH}$, which may be corrected for by performing parallel measurements with $\mathrm{pH}$-sensitive dyes. Another one is possible interference with cellular functions. With current high-end detectors, it is necessary to express $\sim 10 \mu \mathrm{M}$ of the nanosensor to obtain a good signal-to-noise ratio. ${ }^{126}$ The cytosolic concentrations of glucose, glutamate, ATP, lactate, and pyruvate are all much higher, so nanosensor buffering is not a concern for these molecules. NADH is in the nanomolar range ${ }^{120}$ and, therefore, the sensor contributes to the NADH buffering capacity. These and other limitations of the sensors like phototoxicity, toxicity of the fluorescent proteins, and difficulties for subcellular targeting are analyzed elsewhere. $^{78,127,128}$

\section{Concluding Remarks}

Metabolism makes up a large part of what cells are, the sum of a finite number of chemical reactions involving interactions between structural and functional modules at different levels of complexity. In the brain, metabolism is also a player in high-order processes, such as synaptic remodeling, memory, and cognition. In order to tackle the metabolic modules and the interactions between them with a reductionist or systems biology approach, it is necessary to develop new technologies. Genetically encoded nanosensors are noninvasive and can be used from cultured cells to living animals, spanning several metabolic hierarchies. Nanosensors for six metabolites have been validated in mammalian cells, which allow real-time monitoring of relative level and, in some cases, of concentration and flux. A comprehensive study of the metabolic network will require the development of new sensors, most urgently for metabolites involved in glycogen cycling, the pentose phosphate pathway, and the Krebs cycle. Together with the continued improvement in protein expression strategies and new imaging technologies, metabolite nanosensors herald an exciting era of high-resolution characterization of metabolism in the brain and other organs. The relative location within the metabolic network of the six metabolites covered in this review gives some idea of how much remains to be done (Fig. 3).

\section{Acknowledgments}

We thank Dr. Bruno Weber for helpful discussions and Dr. Karen Everett for critical reading of the manuscript. This work was partially funded by Fondecyt 1130095 . The Centro de Estudios Científicos is funded by the Chilean Government through the
Centers of Excellence Basal Financing Program of CONICYT. The recent finding of the lactate receptor HCAR1/GPR81 in the brain strongly contributes to the emerging notion that lactate acts as an intercellular signal in this organ. ${ }^{130}$

\section{References}

1. M. E. Gibbs, D. G. Anderson, and L. Hertz, "Inhibition of glycogenolysis in astrocytes interrupts memory consolidation in young chickens," Glia 54(3), 214-222 (2006).

2. A. Suzuki et al., "Astrocyte-neuron lactate transport is required for longterm memory formation," Cell 144(5), 810-823 (2011).

3. L. A. Newman, D. L. Korol, and P. E. Gold, "Lactate produced by glycogenolysis in astrocytes regulates memory processing," PLoS One 6(12), e28427 (2011).

4. C. Florian et al., "Astrocyte-derived adenosine and A1 receptor activity contribute to sleep loss-induced deficits in hippocampal synaptic plasticity and memory in mice," J. Neurosci. 31(19), 6956-6962 (2011).

5. L. Bozzo, J. Puyal, and J. Y. Chatton, "Lactate modulates the activity of primary cortical neurons through a receptor-mediated pathway," PLoS One 8(8), e71721 (2013).

6. F. Tang et al., "Lactate-mediated glia-neuronal signalling in the mammalian brain," Nature Commun. 5, 3284 (2014).

7. L. F. Barros, "Metabolic signaling by lactate in the brain," Trends Neurosci. 36(7), 396-404 (2013).

8. M. S. Goyal et al., "Aerobic glycolysis in the human brain is associated with development and neotenous gene expression," Cell Metab. 19(1), 49-57 (2014).

9. M. E. Raichle et al., "A default mode of brain function," Proc. Natl. Acad. Sci. U. S. A. 98(2), 676-682 (2001).

10. A. G. Vlassenko et al., "Spatial correlation between brain aerobic glycolysis and amyloid-beta (Abeta) deposition," Proc. Natl. Acad. Sci. U. S. A. 107(41), 17763-17767 (2010).

11. A. W. Bero et al., "Neuronal activity regulates the regional vulnerability to amyloid-beta deposition," Nat. Neurosci. 14(6), 750-756 (2011).

12. K. Fonseca-Azevedo and S. Herculano-Houzel, "Metabolic constraint imposes tradeoff between body size and number of brain neurons in human evolution," Proc. Natl. Acad. Sci. U. S. A. 109(45), 1857118576 (2012).

13. A. R. Peacocke, An Introduction to the Physical Chemistry of Biological Organization, Oxford University Press, Oxford (1983).

14. I. Fernandez-Moncada and L. F. Barros, "Non-preferential fuelling of the Na+/K+ ATPase pump," Biochem. J. [Epub ahead of print] (2014).

15. D. W. Maughan, J. A. Henkin, and J. O. Vigoreaux, "Concentrations of glycolytic enzymes and other cytosolic proteins in the diffusible fraction of a vertebrate muscle proteome," Mol. Cell Proteomics 4(10), 1541-1549 (2005).

16. J. E. Wilson, "Isozymes of mammalian hexokinase: structure, subcellular localization and metabolic function," J. Exp. Biol. 206(12), 2049-2057 (2003).

17. E. N. Genda et al., "Co-compartmentalization of the astroglial glutamate transporter, GLT-1, with glycolytic enzymes and mitochondria," $J$. Neurosci. 31(50), 18275-18288 (2011).

18. I. Llorente-Folch et al., "Calcium-regulation of mitochondrial respiration maintains ATP homeostasis and requires ARALAR/AGC1-malate aspartate shuttle in intact cortical neurons,' J. Neurosci. 33(35), 13957139571 (2013).

19. J. Satrustegui, B. Pardo, and A. del Arco, "Mitochondrial transporters as novel targets for intracellular calcium signaling," Physiol. Rev. 87(1), 29-67 (2007).

20. F. N. Gellerich et al., "The control of brain mitochondrial energization by cytosolic calcium: the mitochondrial gas pedal," IUBMB Life $\mathbf{6 5}(3)$, 180-190 (2013).

21. G. A. Brooks, "Cell-cell and intracellular lactate shuttles," J. Physiol. 587(23), 5591-5600 (2009).

22. A. K. Bouzier-Sore et al., "Competition between glucose and lactate as oxidative energy substrates in both neurons and astrocytes: a comparative NMR study," Eur. J. Neurosci. 24(6), 1687-1694 (2006). 
23. P. Jakoby et al., "Higher transport and metabolism of glucose in astrocytes compared with neurons: a multiphoton study of hippocampal and cerebellar tissue slices," Cereb. Cortex 24(1), 222-231 (2013).

24. D. Lovatt et al., "The transcriptome and metabolic gene signature of protoplasmic astrocytes in the adult murine cortex," J. Neurosci. 27(45), 12255-12266 (2007).

25. J. D. Cahoy et al., "A transcriptome database for astrocytes, neurons, and oligodendrocytes: a new resource for understanding brain development and function," J. Neurosci. 28(1), 264-278 (2008).

26. A. Herrero-Mendez et al., "The bioenergetic and antioxidant status of neurons is controlled by continuous degradation of a key glycolytic enzyme by APC/C-Cdh1," Nat. Cell Biol. 11(6), 747-752 (2009).

27. S. Fernandez-Fernandez, A. Almeida, and J. P. Bolanos, "Antioxidant and bioenergetic coupling between neurons and astrocytes," Biochem. J. 443(1), 3-11 (2012).

28. N. D. Halim et al., "Phosphorylation status of pyruvate dehydrogenase distinguishes metabolic phenotypes of cultured rat brain astrocytes and neurons," Glia 58(10), 1168-1176 (2010).

29. M. Belanger, I. Allaman, and P. J. Magistretti, "Brain energy metabolism: focus on astrocyte-neuron metabolic cooperation," Cell Metab. 14(6), 724-738 (2011).

30. A. K. Bouzier-Sore and L. Pellerin, "Unraveling the complex metabolic nature of astrocytes," Front Cell Neurosci. 7, 179 (2013).

31. I. Ruminot et al.,"NBCe1 mediates the acute stimulation of astrocytic glycolysis by extracellular K+," J. Neurosci. 31(40), 14264-14271 (2011).

32. G. Azarias et al., "Glutamate transport decreases mitochondrial $\mathrm{pH}$ and modulates oxidative metabolism in astrocytes," J. Neurosci. 31(10), 3550-3559 (2011).

33. J. L. Stobart and C. M. Anderson, "Multifunctional role of astrocytes as gatekeepers of neuronal energy supply," Front Cell Neurosci. 7, 38 (2013).

34. S. C. Lange et al., "Primary cultures of astrocytes: their value in understanding astrocytes in health and disease," Neurochem. Res. 37(11), 2569-2588 (2012).

35. A. M. Brennan, J. A. Connor, and C. W. Shuttleworth, "NAD(P)H fluorescence transients after synaptic activity in brain slices: predominant role of mitochondrial function," J. Cereb. Blood Flow Metab. 26(11), 1389-1406 (2006).

36. F. Galeffi et al., "Simultaneous monitoring of tissue PO2 and NADH fluorescence during synaptic stimulation and spreading depression reveals a transient dissociation between oxygen utilization and mitochondrial redox state in rat hippocampal slices," J. Cereb. Blood Flow Metab. 31(2), 626-639 (2011).

37. C. W. Shuttleworth, "Use of $\mathrm{NAD}(\mathrm{P}) \mathrm{H}$ and flavoprotein autofluorescence transients to probe neuron and astrocyte responses to synaptic activation," Neurochem. Int. 56(3), 379-386 (2010).

38. J. Hirrlinger and R. Dringen, "The cytosolic redox state of astrocytes: maintenance, regulation and functional implications for metabolite trafficking," Brain Res. Rev. 63(1-2), 177-188 (2010).

39. K. C. Reinert et al., "Cellular and metabolic origins of flavoprotein autofluorescence in the cerebellar cortex in vivo," Cerebellum 10(3), 585-599 (2011).

40. L. F. Barros et al., "Small is fast: astrocytic glucose and lactate metabolism at cellular resolution," Front Cell Neurosci. 7, 27 (2013).

41. W. H. Kim et al., "Visualizing sweetness: increasingly diverse applications for fluorescent-tagged glucose bioprobes and their recent structural modifications," Sensors 12(4), 5005-5027 (2012).

42. L. F. Barros et al., "Kinetic validation of 6-NBDG as a probe for the glucose transporter GLUT1 in astrocytes," J. Neurochem. 109(1), 94-100 (2009).

43. A. Loaiza, O. H. Porras, and L. F. Barros, "Glutamate triggers rapid glucose transport stimulation in astrocytes as evidenced by real-time confocal microscopy," J. Neurosci. 23(19), 7337-7342 (2003).

44. O. H. Porras, A. Loaiza, and L. F. Barros, "Glutamate mediates acute glucose transport inhibition in hippocampal neurons," J. Neurosci. 24(43), 9669-9673 (2004).

45. J. Chuquet et al., "Predominant enhancement of glucose uptake in astrocytes versus neurons during activation of the somatosensory cortex," $J$. Neurosci. 30(45), 15298-15303 (2010).

46. Y. Bernardinelli, P. J. Magistretti, and J. Y. Chatton, "Astrocytes generate Na+-mediated metabolic waves," Proc. Natl. Acad. Sci. U. S. A. 101(41), 14937-14942 (2004).
47. N. Rouach et al., "Astroglial metabolic networks sustain hippocampal synaptic transmission," Science 322(5907), 1551-1555 (2008).

48. L. F. Barros et al., "Preferential transport and metabolism of glucose in Bergmann glia over Purkinje cells: a multiphoton study of cerebellar slices," Glia 57(9), 962-970 (2009).

49. L. Pellerin and P. J. Magistretti, "Glutamate uptake into astrocytes stimulates aerobic glycolysis: a mechanism coupling neuronal activity to glucose utilization," Proc. Natl. Acad. Sci. U. S. A. 91(22), 1062510629 (1994).

50. L. Pellerin et al., "Activity-dependent regulation of energy metabolism by astrocytes: an update," Glia 55(12), 1251-1262 (2007).

51. A. Miyawaki et al., "Fluorescent indicators for $\mathrm{Ca} 2+$ based on green fluorescent proteins and calmodulin," Nature 388(6645), 882-887 (1997).

52. M. Fehr et al., "In vivo imaging of the dynamics of glucose uptake in the cytosol of COS-7 cells by fluorescent nanosensors," J. Biol. Chem. 278(21), 19127-19133 (2003).

53. H. Takanaga, B. Chaudhuri, and W. B. Frommer, "GLUT1 and GLUT9 as major contributors to glucose influx in HepG2 cells identified by a high sensitivity intramolecular FRET glucose sensor," Biochim. Biophys. Acta 1778(4), 1091-1099 (2008).

54. B. H. Hou et al., "Optical sensors for monitoring dynamic changes of intracellular metabolite levels in mammalian cells," Nat. Protoc. 6(11), 1818-1833 (2011).

55. L. Q. Chen et al., "Sugar transporters for intercellular exchange and nutrition of pathogens," Nature 468(7323), 527-532 (2010).

56. C. X. Bittner et al., "High resolution measurement of the glycolytic rate," Front. Neuroenergetics 2, 1-11 (2010).

57. B. Chaudhuri, F. Hormann, and W. B. Frommer, "Dynamic imaging of glucose flux impedance using FRET sensors in wild-type Arabidopsis plants," J. Exp. Bot. 62(7), 2411-2417 (2011).

58. C. Bermejo et al., "Dynamic analysis of cytosolic glucose and ATP levels in yeast using optical sensors," Biochem. J. 432(2), 399-406 (2010).

59. C. X. Bittner et al., "Fast and reversible stimulation of astrocytic glycolysis by $\mathrm{K}^{+}$and a delayed and persistent effect of glutamate," J. Neurosci. 31, 4709-4713 (2011).

60. H. J. Huber et al., "Glucose metabolism determines resistance of cancer cells to bioenergetic crisis after cytochrome-c release," Mol. Syst. Biol. 7, 470 (2011).

61. G. Grossmann et al., "The RootChip: an integrated microfluidic chip for plant science," Plant Cell 23(12), 4234-4240 (2011).

62. M. Prebil et al., "Dynamic monitoring of cytosolic glucose in single astrocytes," Glia 59(6), 903-913 (2011).

63. G. Grossmann et al., "Time-lapse fluorescence imaging of Arabidopsis root growth with rapid manipulation of the root environment using the RootChip," J. Vis. Exp. (65), e4290 (2012).

64. M. T. Kaminski, S. Lenzen, and S. Baltrusch, "Real-time analysis of intracellular glucose and calcium in pancreatic beta cells by fluorescence microscopy," Biochim. Biophys. Acta 1823(10), 1697-1707 (2012).

65. T. Sotelo-Hitschfeld, I. Fernández-Moncada, and L. F. Barros, "Acute feedback control of astrocytic glycolysis by lactate," Glia 60(4), 674680 (2012).

66. C. Bermejo et al., "Differential regulation of glucose transport activity in yeast by specific cAMP signatures," Biochem. J. 452(3), 489-497 (2013).

67. B. Chaudhuri et al., "Protonophore- and $\mathrm{pH}$-insensitive glucose and sucrose accumulation detected by FRET nanosensors in Arabidopsis root tips," Plant J. 56(6), 948-962 (2008).

68. H. Takanaga and W. B. Frommer, "Facilitative plasma membrane transporters function during ER transit," FASEB J. 24(8), 2849-2858 (2010).

69. M. T. Kaminski et al., "Glucose-induced dissociation of glucokinase from its regulatory protein in the nucleus of hepatocytes prior to nuclear export," Biochim. Biophys. Acta 1843(3), 554-564 (2014).

70. K. Deuschle et al., "Rapid metabolism of glucose detected with FRET glucose nanosensors in epidermal cells and intact roots of Arabidopsis RNA-silencing mutants," Plant Cell 18(9), 2314-2325 (2006).

71. M. Fehr et al., "Live imaging of glucose homeostasis in nuclei of COS-7 cells," J. Fluoresc. 14(5), 603-609 (2004).

72. M. Fehr et al., "Evidence for high-capacity bidirectional glucose transport across the endoplasmic reticulum membrane by genetically encoded fluorescence resonance energy transfer nanosensors," Mol. Cell Biol. 25(24), 11102-11112 (2005). 
73. S. John, J. N. Weiss, and B. Ribalet, "Subcellular localization of hexokinases I and II directs the metabolic fate of glucose," PLoS One 6(3), e17674 (2011).

74. P. B. Kovacic et al., "New insights into cytosolic glucose levels during differentiation of 3T3-L1 fibroblasts into adipocytes," J. Biol. Chem. 286(15), 13370-13381 (2011).

75. G. Calmettes et al., "Hexokinase-mitochondrial interactions regulate glucose metabolism differentially in adult and neonatal cardiac myocytes," J. Gen. Physiol. 142(4), 425-436 (2013).

76. A. Zambon et al., "Determination of glucose flux in live myoblasts by microfluidic nanosensing and mathematical modeling," Integr. Biol. 6(3), 277-288 (2014).

77. M. Kreft et al., "Diffusion of D-glucose measured in the cytosol of a single astrocyte," Cell Mol. Life Sci. 70(8), 1483-1492 (2013).

78. L. F. Barros et al., "Fluorescent nanosensor based flux analysis: overview and the example of glucose," in Springer Protocols: Brain Energy Metabolism, H. S. Waagepetersen and J. Hirrlinger, Eds., Springer, Berlin (2014).

79. H. E. Parker et al., "Predominant role of active versus facilitative glucose transport for glucagon-like peptide-1 secretion," Diabetologia 55(9), 2445-2455 (2012).

80. C. Bermejo et al., "Optical sensors for measuring dynamic changes of cytosolic metabolite levels in yeast," Nat. Protoc. 6(11), 1806-1817 (2011).

81. M. Prebil et al., "Changes in cytosolic glucose level in ATP stimulated live astrocytes," Biochem. Biophys. Res. Commun. 405(2), 308-313 (2011).

82. S. Okumoto, H. Takanaga, and W. B. Frommer, "Quantitative imaging for discovery and assembly of the metabo-regulome," New Phytol. 180(2), 271-295 (2008).

83. S. Okumoto et al., "Detection of glutamate release from neurons by genetically encoded surface-displayed FRET nanosensors," Proc. Natl. Acad. Sci. U. S. A 102(24), 8740-8745 (2005).

84. S. A. Hires, Y. Zhu, and R. Y. Tsien, "Optical measurement of synaptic glutamate spillover and reuptake by linker optimized glutamate-sensitive fluorescent reporters," Proc. Natl. Acad. Sci. U. S. A. 105(11), 4411-4416 (2008).

85. K. Deuschle et al., "Construction and optimization of a family of genetically encoded metabolite sensors by semirational protein engineering," Protein Sci. 14(9), 2304-2314 (2005).

86. C. Dulla et al., "Imaging of glutamate in brain slices using FRET sensors," J. Neurosci. Methods 168(2), 306-319 (2008).

87. C. V. Melo et al., "Spatiotemporal resolution of BDNF neuroprotection against glutamate excitotoxicity in cultured hippocampal neurons," Neuroscience 237, 66-86 (2013).

88. A. Firl et al., "Extrasynaptic glutamate and inhibitory neurotransmission modulate ganglion cell participation during glutamatergic retinal waves," J. Neurophysiol. 109(7), 1969-1978 (2013).

89. J. S. Marvin et al., "An optimized fluorescent probe for visualizing glutamate neurotransmission," Nat. Methods 10(2), 162-170 (2013).

90. B. G. Borghuis et al., "Two-photon imaging of nonlinear glutamate release dynamics at bipolar cell synapses in the mouse retina," $J$. Neurosci. 33(27), 10972-10985 (2013).

91. H. Imamura et al., "Visualization of ATP levels inside single living cells with fluorescence resonance energy transfer-based genetically encoded indicators," Proc. Natl. Acad. Sci. U. S. A 106(37), 15651-15656 (2009).

92. M. U. De, C. Castelbou, and N. Demaurex, "Uncoupling protein 3 (UCP3) modulates the activity of Sarco/endoplasmic reticulum $\mathrm{Ca} 2$ +-ATPase (SERCA) by decreasing mitochondrial ATP production," J. Biol. Chem. 286(37), 32533-32541 (2011).

93. T. Tsuyama et al., "In vivo fluorescent adenosine 5'-triphosphate (ATP) imaging of Drosophila melanogaster and Caenorhabditis elegans by using a genetically encoded fluorescent ATP biosensor optimized for low temperatures," Anal. Chem. 85(16), 7889-7896 (2013).

94. N. Hatsugai et al., "Changes in cytosolic ATP levels and intracellular morphology during bacteria-induced hypersensitive cell death as revealed by real-time fluorescence microscopy imaging," Plant Cell Physiol. 53(10), 1768-1775 (2012).

95. H. Kioka et al., "Evaluation of intramitochondrial ATP levels identifies G0/G1 switch gene 2 as a positive regulator of oxidative phosphorylation," Proc. Natl. Acad. Sci. U. S. A. 111(1), 273-278 (2014).
96. M. Waldeck-Weiermair et al., "Leucine zipper EF hand-containing transmembrane protein 1 (Letm1) and uncoupling proteins 2 and 3 (UCP2/3) contribute to two distinct mitochondrial Ca2+ uptake pathways," J. Biol. Chem. 286(32), 28444-28455 (2011).

97. T. Ando et al., "Visualization and measurement of ATP levels in living cells replicating hepatitis C virus genome RNA," PLoS Pathog. 8(3), e1002561 (2012).

98. A. G. Barsukova, D. Bourdette, and M. Forte, "Mitochondrial calcium and its regulation in neurodegeneration induced by oxidative stress," Eur. J. Neurosci. 34(3), 437-447 (2011).

99. I. Goehring et al., "Plasma membrane potential oscillations in insulin secreting Ins-1 832/13 cells do not require glycolysis and are not initiated by fluctuations in mitochondrial bioenergetics," J. Biol. Chem. 287(19), 15706-15717 (2012).

100. J. Kishikawa et al., "MRT letter: expression of ATP sensor protein in Caenorhabditis elegans," Microsc. Res. Tech. 75(1), 15-19 (2012).

101. M. Fujikawa et al., "Assessing actual contribution of IF1, inhibitor of mitochondrial FoF1, to ATP homeostasis, cell growth, mitochondrial morphology, and cell viability," J. Biol. Chem. 287(22), 18781-18787 (2012).

102. J. Uchi et al., "Overexpression of ryanodine receptor type 1 enhances mitochondrial fragmentation and $\mathrm{Ca} 2+$-induced ATP production in cardiac H9c2 myoblasts," Am. J. Physiol. Heart Circ. Physiol. 305(12), H1736-H1751 (2013).

103. C. M. Dalton, G. Szabadkai, and J. Carroll, "Measurement of ATP in single oocytes: impact of maturation and cumulus cells on levels and consumption," J. Cell Physiol. 229(3), 353-361 (2014).

104. J. Krajnakova et al., "Changes in ATP, glucose-6-phosphate and NAD (P)H cellular levels during the proliferation and maturation phases of Abies alba Mill. embryogenic cultures," Tree Physiol. 33(10), 1099_ 1110 (2013).

105. J. Toloe et al., "Metabolic differences in hippocampal 'Rett' neurons revealed by ATP imaging," Mol. Cell Neurosci. 13, 47-56 (2014).

106. L. Menger et al., "Cardiac glycosides exert anticancer effects by inducing immunogenic cell death," Sci. Transl. Med. 4(143), 143 ra99 (2012).

107. M. Nakano et al., " $\mathrm{Ca}(2)(+)$ regulation of mitochondrial ATP synthesis visualized at the single cell level," ACS Chem. Biol. 6(7), 709-715 (2011).

108. H. J. Kwon et al., "Synchronized ATP oscillations have a critical role in prechondrogenic condensation during chondrogenesis," Cell Death Dis. 3, e278 (2012).

109. A. Roesch et al., "Overcoming intrinsic multidrug resistance in melanoma by blocking the mitochondrial respiratory chain of slow-cycling JARID1B(high) cells," Cancer Cell 23(6), 811-825 (2013).

110. Y. Gouriou, P. Bijlenga, and N. Demaurex, "Mitochondrial Ca2+ uptake from plasma membrane Cav3.2 protein channels contributes to ischemic toxicity in PC12 cells," J. Biol. Chem. 288(18), 1245912468 (2013).

111. F. Valsecchi et al., "Metabolic consequences of NDUFS4 gene deletion in immortalized mouse embryonic fibroblasts," Biochim. Biophys. Acta 1817(10), 1925-1936 (2012).

112. D. C. Liemburg-Apers et al., "Quantitative glucose and ATP sensing in mammalian cells," Pharm. Res. 28(11), 2745-2757 (2011).

113. J. Berg, Y. P. Hung, and G. Yellen, "A genetically encoded fluorescent reporter of ATP:ADP ratio," Nat. Methods 6(2), 161-166 (2009).

114. M. Tantama et al., "Imaging energy status in live cells with a fluorescent biosensor of the intracellular ATP-to-ADP ratio," Nat. Commun. 4, 2550 (2013).

115. A. I. Tarasov et al., "The mitochondrial Ca2+ uniporter MCU is essential for glucose-induced ATP increases in pancreatic beta-cells," PLoS One 7(7), e39722 (2012).

116. Q. Zhang et al., "Role of KATP channels in glucose-regulated glucagon secretion and impaired counterregulation in type 2 diabetes," Cell Metab. 18(6), 871-882 (2013).

117. D. Zala et al., "Vesicular glycolysis provides on-board energy for fast axonal transport," Cell 152(3), 479-491 (2013).

118. Y. P. Hung et al., "Imaging cytosolic NADH-NAD(+) redox state with a genetically encoded fluorescent biosensor," Cell Metab. 14(4), 545554 (2011). 
119. D. S. Bilan et al., "Genetically encoded fluorescent indicator for imaging $\mathrm{NAD}(+) / \mathrm{NADH}$ ratio changes in different cellular compartments," Biochim. Biophys. Acta 1840(3), 951-957 (2014).

120. Y. Zhao et al., "Genetically encoded fluorescent sensors for intracellular NADH detection," Cell Metab. 14(4), 555-566 (2011).

121. A. S. Martín et al., "A genetically encoded FRET lactate sensor and its use to detect the Warburg effect in single cancer cells," PLoS One 8(2), e57712 (2013).

122. A. S. Martín et al., "Imaging mitochondrial flux in single cells with a FRET sensor for pyruvate," PLoS One 9(1), e85780 (2014).

123. S. Cerdan et al., "The redox switch/redox coupling hypothesis," Neurochem. Int. 48(6-7), 523-530 (2006).

124. L. Pelkmans, "Cell biology. Using cell-to-cell variability-a new era in molecular biology," Science 336(6080), 425-426 (2012).

125. R. Zenobi, "Single-cell metabolomics: analytical and biological perspectives," Science 342(6163), 1243259 (2013).
126. A. Miyawaki et al., "Dynamic and quantitative $\mathrm{Ca} 2+$ measurements using improved cameleons," Proc. Natl. Acad. Sci. U. S. A 96(5), 2135-2140 (1999).

127. R. De Michele, F. Carimi, and W. B. Frommer, "Mitochondrial biosensors," Int. J. Biochem. Cell Biol. 48, 39-44 (2014).

128. T. Mathew, Y. P. Hung, and G. Yellen, "Optogenetic reporters: fluorescent protein-based genetically encoded of signaling and metabolism in the brain," Chapter 12 in Progress in Brain Research, T. Knopfel and E. Boyden, Eds., pp. 235-263, Elsevier, Amsterdam (2012).

129. S. A. Kauffman, The Origins of Order, Oxford University Press, New York \& Oxford (1993).

130. K. H. Lauritzen et al., "Lactate receptor sites link neurotransmission, neurovascular coupling, and brain energy metabolism," Cereb. Cortex (2013) [Epub ahead of print].

Biographies of the authors are not available. 